\title{
Methods and constraints for the correction of the error beam pick-up in single dish radio observations
}

\author{
F. Bensch ${ }^{1}$, J. Stutzki ${ }^{1}$, and A. Heithausen ${ }^{1,2}$ \\ 1 I. Physikalisches Institut der Universität zu Köln, Zülpicher Straße 77, 50937 Köln, Germany \\ 2 Radioastronomisches Institut der Universität Bonn, Auf dem Hügel 71, 53121 Bonn, Germany
}

Received 27 January 2000 / Accepted 1 August 2000

\begin{abstract}
The beam pattern of a single dish radio telescope is given by the main beam and additional components at larger angles, usually called error beam or stray pattern. The latter have relatively small peak amplitudes (typ. below $-25 \mathrm{~dB}$ ), depending on the rms surface error of the primary reflector. However, because of their large angular extent, they are sensitive to extended sources, and a significant fraction of the observed intensity can result from error beam pick-up. For (sub-)mm observations suffering from error beam pick-up we introduce a new temperature scale for the corrected data, the corrected main beam brightness temperature $T_{\mathrm{mb}, \mathrm{c}}$, which provides a better approximation to the intensity detected by the main beam than the commonly used antenna temperature and main beam brightness temperature. We consider two different correction methods. The first method uses complementary observations obtained with a smaller telescope. Smeared to the angular resolution of the error beam pattern they provide an estimate of the error beam pick-up in the observations of the large telescope. For the second method, the error beam pick-up is de-convolved from the observed map in Fourier space. The requirements for both correction methods and their advantages and limitations are discussed in detail. Both correction methods require additional observations, unless the full spatial extent of the emission is observed. We find that the deconvolution method is attractive for the correction of fully sampled maps with an angular extent much larger than the error beam pattern. For smaller maps and more sparsely sampled observations, the subtraction method is favorable, because the additional observations with a small telescope are less time consuming.
\end{abstract}

Key words. methods: data analysis - techniques: miscellaneous - telescopes - radio lines: ISM

\section{Introduction}

The presence of a significant error beam pick-up was noted for HI observations more than three decades ago (cf. van Woerden et al. 1962; Westerhout et al. 1973). The error or stray pattern responsible for the additional pick-up mainly resulted from surface irregularities in the primary reflector (cf. Harten 1973; Heiles \& Hoffman 1968). The improved reflector surface of present day $\mathrm{cm}$ wave telescopes greatly reduced the amplitude of these components and the residual error beam is often found to be dominated by spillover and scattering. Typically, these components are not axially-symmetric with amplitudes below -30 dB (cf. Kalberla et al. 1980).

With the increasing number of large telescopes and sensitive receivers for the (sub-) $\mathrm{mm}$ range, the importance of an error beam correction was recognized for observations at shorter wavelengths (cf. Schneider et al. 1998).

Send offprint requests to: F. Bensch, e-mail: bensch@ph1.uni-koeln.de
Essential pre-requisite for a correction is an accurately measured beam pattern. For the IRAM $30 \mathrm{~m}$ telescope, this was done by Garcia-Burillo et al. (1993) and Greve et al. (1998). Based on these studies, Bensch et al. (2001) determined the influence of the error beam pick-up in the data set of the IRAM key-project, and estimated the accuracy of the applied correction method. They demonstrate that the error beam pick-up of the IRAM $30 \mathrm{~m}$ at wavelengths of $1.3 \mathrm{~mm}$ can account for 20 $50 \%$ of the observed intensity (map average), and locally for up to $100 \%$.

The error beam problem is well known to HI observers and large surveys are routinely corrected for the additional pick-up (cf. Hartmann et al. 1996). For observations at (sub-)mm wavelengths, however, only very few quantitative studies exist (cf. Garcia-Burillo et al. 1993; Dame \& Thaddeus 1994; Schneider et al. 1998), despite the fact the error beam problem is expected to be quite common also to large (sub-)mm telescopes. In addition, the ratio of the error beam pattern to the spatial extent of the source can be very different for $\mathrm{cm}$ and (sub-)mm wavelengths. The correction methods and results found 
for HI observations therefore do not necessarily apply to the observations made at shorter wavelengths, and a more detailed study of the influence of error beam pick-up and the correction methods is needed.

This paper supplements Paper III in the series presenting the results of the IRAM key-project "Small-scale structure of pre-star forming regions". The data set of the key-project and first results are published by Falgarone et al. (1998, Paper I) and Heithausen et al. (1998). The data reduction is presented by Panis et al. (in preparation; Paper II) and the influence and correction of the error beam pick-up is discussed by Bensch et al. (2001, Paper III). The present paper puts the error beam correction of (sub-)mm observations on firmer grounds. The relevant definitions are provided and the possible correction methods are reviewed, discussing their advantages and limitations. In addition, a guideline is provided for observers who wish to correct their own data.

In Sect. 2, we introduce the notation and give the definition of the corrected main beam brightness temperature. The error beam pattern of (sub-)mm telescopes is discussed in Sect. 3, and the error beam correction methods are presented in Sects. 4 and 5. The requirements of both correction methods are discussed and compared, their advantages and limitations are outlined (Sect. 6). A summary is given in Sect. 7 .

\section{Definitions}

In the following, we review some of the definitions and basic relations which are relevant for the intensity calibration of radio astronomical single dish observations. For a more detailed presentation we refer to the publications of e.g. Baars (1973) and Rohlfs \& Wilson (1996). The calibration of observations at (sub-)mm wavelengths is covered by e.g. Kutner \& Ulich (1981), Downes (1989) and Kramer (1997).

Consider a radio telescope pointing toward a source of brightness distribution $T_{\mathrm{b}}(\theta, \phi)=T_{\mathrm{b}} \cdot S(\theta, \phi)$, where $T_{\mathrm{b}}$ is the peak brightness temperature, and $S(\theta, \phi)$ varies between 0 and 1 . The observed antenna temperature, corrected for atmospheric and rear-ward losses, then reads

$$
\begin{aligned}
T_{\mathrm{A}}^{*} & =T_{\mathrm{b}} \frac{\iint_{\text {source }} P(\theta, \phi) S\left(\theta-\theta_{0}, \phi-\phi_{0}\right) \mathrm{d} \Omega}{\Omega_{2 \pi}} \\
& =T_{\mathrm{b}} \frac{P * S}{\Omega_{2 \pi}},
\end{aligned}
$$

where $P(\theta, \phi)$ is the antenna pattern of the telescope (normalization $P(0,0)=1)$. Here, we have used the "*" symbol as shorthand notation for convolution. Note, that all quantities such as efficiencies, source brightness distribution, etc. depend on the observed frequency, although not further indicated in the following.

The main beam brightness temperature (cf. Downes $1989)$ is defined by $T_{\mathrm{mb}}=\left(F_{\text {eff }} / B_{\text {eff }}\right) T_{\mathrm{A}}^{*}$, where $F_{\text {eff }} / B_{\text {eff }}=\Omega_{2 \pi} / \Omega_{\mathrm{mb}}$ and $\Omega_{2 \pi}\left(\Omega_{\mathrm{mb}}\right)$ is the integral of the beam pattern $P(\theta, \phi)$ over the forward hemisphere (main beam) of the telescope. The main beam is defined e.g. as the beam pattern enclosed by the $-20 \mathrm{~dB}$ threshold, or the equivalent Gaussian with a half power beam width (HPBW) determined from cross-scans on calibration sources (e.g. planets). Here, we accept the former definition: $P_{\mathrm{mb}}(\theta, \phi)=P(\theta, \phi)$ for $\theta, \phi$ inside the $-20 \mathrm{~dB}$ level, and $P_{\mathrm{mb}}(\theta, \phi)=0$ elsewhere. The forward beam pattern outside the main beam, $P_{2 \pi}-P_{\mathrm{mb}}$, is then denoted as error beam. Note, that this definition of the main beam is not identical to the diffraction beam of a telescope with a perfect reflector surface, because components resulting from surface irregularities partly contribute to $P_{\mathrm{mb}}$. However, this contribution is small in most cases (Sect. 3).

For telescopes with a negligible error beam, the main beam brightness temperature gives the intensity detected by the main beam, averaged over the solid angle of the main beam, $\Omega_{\mathrm{mb}}$. For telescopes where a significant error beam is present, the main beam brightness temperature potentially overestimates the intensity detected by the main beam, whereas the antenna temperature underestimates it. Both, Kutner \& Ulich (1981) and Downes (1989), discuss the consequences of a non-negligible error beam pickup. Kutner \& Ulich (1981) a priori define a temperature scale $T_{\mathrm{R}}^{*}$ which gives the intensity averaged over the "diffraction pattern of the telescope", with the components of the error beam pattern (resulting from surface irregularities) being included. Pick-up from larger angles (due to e.g. spillover past the secondary) are included in the a priori unknown source coupling efficiency $\eta_{\mathrm{c}}$. Downes (1989) points out that in case of a significant error beam, the main beam efficiency $B_{\text {eff }}$ has to be replaced by the larger full beam efficiency, encompassing the main lobe and the error beam pattern. The resulting quantity is then denoted as beam averaged brightness temperature.

These definitions avoid the problem that the main beam-averaged intensity is exceedingly overestimated because of the additional error beam pick-up. The main draw back is, however, that the thus defined quantities depend on the details of the error beam pattern. In addition, the error beam pick-up might give rise to "fake" velocity and spatial features, depending on the angular extent and the velocity structure of the source. Therefore, a simple scaling of the efficiencies cannot properly correct for the error beam pick-up. This motivates us to introduce a new quantity, the corrected main beam brightness temperature,

$T_{\mathrm{mb}, \mathrm{c}}=T_{\mathrm{b}} \frac{P_{\mathrm{mb}} * S}{\Omega_{\mathrm{mb}}}=\frac{F_{\mathrm{eff}}}{B_{\mathrm{eff}}} T_{\mathrm{b}} \frac{P_{\mathrm{mb}} * S}{\Omega_{2 \pi}}$,

where we use the above definition of the main beam, and the convolution integral extends over the forward hemisphere of the telescope. The crucial point is, however, not the definition of a new temperature scale, but to find a correction method which is not too time consuming and which can be applied to observed data with the limited observing time, limited signal-to-noise ratio, and the data reduction resources available.

For cm wave telescopes, an equivalent quantity is introduced by Kalberla et al. (1980) with the corrected brightness temperature $T_{\mathrm{C}}$. In fact, both $\left(T_{\mathrm{C}}\right.$ and $\left.T_{\mathrm{mb}, \mathrm{c}}\right)$ 
are identical if the same main beam definition is used. However, the main beam definition for $\mathrm{cm}$ and (sub-) $\mathrm{mm}$ telescopes often differ because of practical reasons. Due to the limited signal-to-noise ratio of the observations at (sub-)mm wavelengths, the dynamical range in intensity often is not sufficient to accurately measure the side-lobes of the beam pattern. Therefore, the main beam definition commonly used for $\mathrm{cm}$ telescopes (beam pattern between first nulls, cf. Hartmann et al. 1996) often cannot be applied to (sub-)mm observations. This motivates us to introduce $T_{\mathrm{mb}, \mathrm{c}}$ as a new quantity for the (sub-)mm range.

With the observed intensity given in terms of antenna temperature $T_{\mathrm{A}}^{*}$ (Eq. (1)), the corrected main beam brightness temperature can be written as

$$
\begin{aligned}
T_{\mathrm{mb}, \mathrm{c}} & =\frac{F_{\mathrm{eff}}}{B_{\mathrm{eff}}}\left[T_{\mathrm{A}}^{*}-T_{\mathrm{b}} \frac{\left(P_{2 \pi}-P_{\mathrm{mb}}\right) * S}{\Omega_{2 \pi}}\right] \\
& =\frac{F_{\mathrm{eff}}}{B_{\mathrm{eff}}}\left[T_{\mathrm{A}}^{*}-T_{\mathrm{eb}}\right]
\end{aligned}
$$

where the convolution integral extends over the forward hemisphere, and $T_{\mathrm{eb}}$ is denoted as error beam pick-up. The error beam correction according to Eq. (3) involves a far more complicated procedure than the calibration of the observations using the main beam efficiency. It requires a detailed knowledge of the beam pattern and information on the spatial distribution of the emission at larger angular scales, and thus additional observations in many cases.

\section{The error beam pattern of single dish radio telescopes}

The error beam pattern arises from imperfections in the primary reflector, spillover past the secondary mirror and scattering from support legs and the secondary mirror. The latter results in a not axially-symmetric error beam and is expected to play a role for telescopes with a primary reflector surface of high accuracy where the surface errors are negligible compared to the wavelength of the observations. Their correction requires information on the actual orientation of the error beam on the sky (cf. Kalberla et al. 1980; Dame \& Thaddeus 1994; Hartmann et al. 1996).

For larger (sub-)mm wave telescopes, the error beam pattern often is dominated by surface errors arising from the limited alignment accuracy of the panels in the primary reflector. The resulting error beam pattern has an approximately Gaussian profile, centered on the main beam, with a HPBW corresponding to the correlation length of the surface error distribution (antenna tolerance theory, see Scheffler 1962; Ruze 1952, 1966; Shifrin 1971; Baars 1973).

Detailed studies of the beam pattern for several large (sub-)mm wave telescopes have shown that the error beam can be represented by a combination of several Gaussian error beams, arising from independent surface error distributions. Examples are the Itatepinga $14 \mathrm{~m}$ telescope (Kaufmann et al. 1987), the JCMT $15 \mathrm{~m}$ (Hills \& Richter 1992; Prestage 1993), and the IRAM
$30 \mathrm{~m}$ (Garcia-Burillo et al. 1993; Greve et al. 1998). The correlation length of the error distributions is given by the linear dimension of the panels, panel frames, and the mounting of the panels on the back-structure of the telescope (e.g. the array of adjustment screws). The similar design of large telescopes, and because the root mean square (rms) of the surface error is not negligible compared to the wavelength for (sub-)mm observations (as opposite to most of the present day $\mathrm{cm}$-wave telescopes), it is reasonable to assume that the error beam pattern is dominated by one (or several) Gaussian components,

$P_{2 \pi}-P_{\mathrm{mb}} \approx \sum_{i} P_{\mathrm{e}, i} \approx \sum_{i} w_{\mathrm{e}, i} G_{\theta_{\mathrm{e}, i}}$,

where $G_{\theta_{\mathrm{e}, i}}=4 \ln 2 /\left(\theta_{\mathrm{e}, i}^{2} \pi\right) \exp \left[-4 \ln 2\left(\theta / \theta_{\mathrm{e}, i}\right)^{2}\right]$ and $w_{\mathrm{e}, i}=\int P_{\mathrm{e}, i} \mathrm{~d} \Omega$. The panels (panel frames) of e.g. the IRAM $30 \mathrm{~m}$ have a linear dimension of typically $\frac{1}{15}$ to $\frac{1}{80}$ the diameter of the primary reflector, and thus the error beam HPBW $\theta_{\mathrm{e}, i}$ is $15-80$ times the main beam HPBW. The amplitude of the Gaussian error beam depends on the ratio of the rms surface error to the wavelength.

Equation (4) is only an approximation to $P_{\mathrm{mb}}-P_{2 \pi}$ and includes two assumptions. Firstly, the Gaussian error beams do not significantly contribute to the integral of the beam pattern on the solid angle covered by the main beam, $\Omega_{\mathrm{mb}}$. Secondly, the contribution of the tapered Airy pattern $\mathcal{A}_{\mathrm{T}}$ outside the main beam is small $\left(\mathcal{A}_{\mathrm{T}}\right.$ is the beam pattern of a telescope with a perfect reflector surface, with no blockage and spillover). The error introduced with both approximations is negligible in most cases. We choose the IRAM $30 \mathrm{~m}$ to give an example. For wavelengths of $\lambda \sim 1.3 \mathrm{~mm}$, the ratio $\left(\iint_{\mathrm{mb}} \sum_{i} P_{\mathrm{e}, i} \mathrm{~d} \Omega\right) /\left(\iint_{\mathrm{mb}} \mathcal{A}_{\mathrm{T}} \mathrm{d} \Omega\right)$ is $\sim 5 \%$, while the contribution of the tapered Airy pattern to the integrated beam pattern outside the $-20 \mathrm{~dB}$ contour is less than one percent, assuming a primary reflector illumination with a $-13 \mathrm{~dB}$ edge taper.

\section{Error beam correction by using observations at lower angular resolution}

With an error beam pattern given by a combination of several Gaussians, the pick-up therein is approximated by

$T_{\mathrm{eb}} \approx T_{\mathrm{b}} \sum_{i} \frac{P_{\mathrm{e}, i} * S}{\Omega_{2 \pi}}=T_{\mathrm{b}} \sum_{i} p_{\mathrm{e}, i}\left[G_{\theta_{\mathrm{e}, i}} * S\right]$,

where $p_{\mathrm{e}, i}$ is the normalized, integrated power in the $i$ th Gaussian, $p_{\mathrm{e}, i}=w_{\mathrm{e}, i} / \Omega_{2 \pi}$. $T_{\mathrm{eb}}$ can be determined using observations made with a smaller telescope, provided that the main beam HPBW of the small telescope $\left(\theta_{\mathrm{mb}}^{\prime}\right)$ is smaller than the HPBW of the error beam, $\theta_{\mathrm{mb}}^{\prime} \leq$ $\theta_{\mathrm{e}, i}$ (in the following, the "prime" denotes quantities of the smaller telescope). With $T_{\mathrm{mb}}^{\prime}$ being the main beam brightness temperature observed with the smaller telescope we have

$T_{\mathrm{eb}} \approx \sum_{i} \frac{w_{i}}{\Omega_{2 \pi}}\left\langle T_{\mathrm{mb}}^{\prime}\right\rangle_{i}$, 
where $\left\langle T_{\mathrm{mb}}^{\prime}\right\rangle_{i}=G_{\Theta_{i}^{\prime}} * T_{\mathrm{mb}}^{\prime}$ denotes the smoothing of the spatial intensity distribution to the angular resolution of the $i$ th error beam $\left(G_{\Theta_{i}^{\prime}}\right.$ is a Gaussian of HPBW $\Theta_{i}^{\prime}=$ $\left.\left(\theta_{\mathrm{e}, i}^{2}-\theta_{\mathrm{mb}}^{\prime 2}\right)^{1 / 2}\right)$. We refer to this method as the (error beam) subtraction method.

\subsection{Requirements for the observations with the smaller telescope}

The accuracy of the error beam correction depends on the accuracy of the beam pattern parameters $\left(\theta_{\mathrm{e}, i}, p_{\mathrm{e}, i}\right)$. For a reliable correction, additional requirements apply to the signal to noise ratio and the spatial extent of the additional observations. For a quantification, we consider a rectangular map $a \times b$, observed with a large telescope. The main beam HPBW of the large large telescope is $\theta_{\mathrm{mb}}$, and the main beam (forward) efficiency is $B_{\text {eff }}\left(F_{\text {eff }}\right.$, respectively). The observed spectra are contaminated by the pick-up in one Gaussian error beam of HPBW $\theta_{\text {eb }}$ and "error beam efficiency" $B_{\mathrm{eb}}=w_{\mathrm{eb}} / \Omega_{4 \pi}$. The signal to noise ratio of the observations is $S N R=\left(T_{\mathrm{A}}^{*} / \Delta T_{\mathrm{A}}^{*}\right)$.

For an error beam correction, additional observations are made with a smaller telescope (main beam and forward efficiency of $B_{\text {eff }}^{\prime}$ and $F_{\text {eff }}^{\prime}$ ). These observations have to cover a larger area $\left(a+2 \Delta^{\prime}\right) \times\left(b+2 \Delta^{\prime}\right)$ because a part of the pick-up originates from outside the map observed with the larger telescope. An estimate for $\Delta^{\prime}$ is given in Appendix A with Eq. (A.4),

$\Delta^{\prime} \gtrsim\left[\frac{\Theta^{\prime 2}}{4 \ln 2} \ln \left(\frac{S N R}{\sqrt{2 \delta_{\mathrm{s}}}} \frac{F_{\mathrm{eff}}^{\prime}}{F_{\mathrm{eff}}} \frac{B_{\mathrm{eb}}}{B_{\mathrm{eff}}^{\prime}}\right)\right]^{1 / 2}$,

where $\Theta^{\prime 2}=\theta_{\mathrm{eb}}^{2}-\theta_{\mathrm{mb}}^{\prime 2}$. Here, $\delta_{\mathrm{s}}$ denotes the fraction of the error beam pick-up which is missed due to the finite angular extent of the map, typically accepted to be of the order of a few percent. Note, that this estimate applies to a uniform intensity distribution of the source. A spatially more extended map (larger $\Delta^{\prime}$ ) is required if strong emission is found nearby, and a smaller $\Delta^{\prime}$ is sufficient for a more compact source distribution.

The error beam subtraction will increase the rms noise of the corrected spectra because of the noise in the observations made with the smaller telescope. Therefore, the rms noise of the latter observations has to be sufficiently small in order to not significantly influence the corrected spectra. With $\Delta T_{\mathrm{A}}^{*}$ being the rms noise of the original (uncorrected) observations, we define the rms noise of the corrected spectra as $\Delta T_{\mathrm{mb}, \mathrm{c}}=\left(1+\delta_{\mathrm{n}}\right)\left(F_{\mathrm{eff}} / B_{\mathrm{eff}}\right) \Delta T_{\mathrm{A}}^{*}$ (Appendix A) and require $\delta_{\mathrm{n}}$ to be small (of the order of a few percent). An upper limit to the required rms noise level of the observations with the small telescope is given by Eq. (A.2),

$\Delta T_{\mathrm{A}}^{* \prime}<\sqrt{\frac{\pi \delta_{\mathrm{n}}}{\ln 2}} \frac{F_{\mathrm{eff}}}{F_{\mathrm{eff}}^{\prime}} \frac{B_{\mathrm{eff}}^{\prime}}{B_{\mathrm{eb}}} \frac{\Theta^{\prime}}{\Delta x^{\prime}}\left(\Delta T_{\mathrm{A}}^{*}\right), \quad \Theta^{\prime}>0$,

where $\Delta x^{\prime}$ is the sampling in the observed map.
In the case of matching HPBWs (of the small telescope's main and the larger telescope's error beam, i.e. $\lim _{\Theta^{\prime} \rightarrow 0}$ ) we find $\Delta^{\prime}=0$ and

$\Delta T_{\mathrm{A}}^{* \prime}<\sqrt{2 \delta_{\mathrm{n}}} \frac{F_{\text {eff }}}{F_{\text {eff }}^{\prime}} \frac{B_{\text {eff }}^{\prime}}{B_{\text {eb }}}\left(\Delta T_{\mathrm{A}}^{*}\right)$.

Note, that the 2-dimensional Gaussian $G_{\Theta^{\prime}}$ with $\Theta^{\prime}>0$ is used to deduce Eq. (8), whereas in the limiting case of $\Theta^{\prime} \rightarrow 0$ we have to consider that $G_{\Theta^{\prime}}$ approaches the $\delta$-function (see Appendix A).

\subsection{Time estimate for the additional observations}

The time needed to obtain the observations with the smaller telescope is $t_{\text {add }}^{\prime} \propto N_{\text {add }}^{\prime}\left(\Delta T_{\mathrm{A}}^{* \prime}\right)^{-2}$, where $N_{\text {add }}^{\prime}=$ $\left(a+2 \Delta^{\prime}\right)\left(b+2 \Delta^{\prime}\right) /\left(\Delta x^{\prime}\right)^{2}$ is the number of positions in the map. In the following we assume fully sampled observations, usually accepted to be fulfilled with a sampling of $\Delta x^{\prime}=0.5 \theta_{\mathrm{mb}}^{\prime}$. We then have

$t_{\mathrm{add}}^{\prime} \propto\left[\frac{\left(a+2 \Delta^{\prime}\right)\left(b+2 \Delta^{\prime}\right)}{\left(\theta_{\mathrm{mb}}^{\prime} / 2\right)^{2}}\left(\Delta T_{\mathrm{A}}^{* \prime}\right)^{-2}\right]$,

where $t_{\text {add }}^{\prime}$ not only depends on $\Delta^{\prime}$ and $\Delta T_{\mathrm{A}}^{* \prime}$, but also on the geometry of the map to be corrected.

For an illustration, we assume that the $N$ positions in the map observed with the large telescope cover a square $(a=b)$. In addition, we assume equal forward efficiencies for both telescopes, $F_{\text {eff }}^{\prime}=F_{\text {eff }}$, and the same equipment and atmospheric conditions for all observations. Dividing Eq. (9) by the time needed to complete the observations in the original map, $t_{\text {map }} \propto N\left(\Delta T_{\mathrm{A}}^{*}\right)^{-2}$, gives

$$
\begin{aligned}
& t_{\mathrm{add}}^{\prime} / t_{\mathrm{map}}=\frac{\ln 2}{\pi \delta_{\mathrm{n}}}\left(\frac{B_{\mathrm{eb}}}{B_{\mathrm{mb}}^{\prime}}\right)^{2} \\
& \times N^{-1}\left[\frac{a}{\Theta^{\prime}}+\left(\frac{1}{\ln 2} \ln \left(\frac{S N R}{\left(2 \delta_{\mathrm{s}}\right)^{1 / 2}} \frac{B_{\mathrm{eb}}}{B_{\mathrm{mb}}^{\prime}}\right)\right)^{1 / 2}\right]^{2},
\end{aligned}
$$

where we have used Eqs. (7) and (8) for $\Delta^{\prime}$ and $\Delta T_{\mathrm{A}}^{* \prime}$.

For an order of magnitude estimate we choose $\delta_{\mathrm{s}}, \delta_{\mathrm{n}}=$ 0.02 and a signal-to-noise ratio of $S N R=10$. In addition, we choose $\left(B_{\mathrm{eb}} / B_{\text {eff }}^{\prime}\right)=0.5$, which is appropriate e.g. for the IRAM $30 \mathrm{~m}$ at $230 \mathrm{GHz}$. In addition, we assume fully sampled observations for the large telescope, $N=a^{2} /\left(\theta_{\mathrm{mb}} / 2\right)^{2}$. This gives

$t_{\text {add }}^{\prime} / t_{\text {map }}=2.76 N^{-1}\left[\frac{N^{1 / 2}}{2 R_{1}}+2.16\right]^{2}$,

which is illustrated in Fig. 1 for different ratios $R_{1}=$ $\sqrt{\theta_{\mathrm{eb}}^{2}-\theta_{\mathrm{mb}}^{\prime 2}} / \theta_{\mathrm{mb}}$.

In order to give a numerical example and to compare the results obtained here to those obtained for a deconvolution of the error beam pattern in Fourier space (Sect. 5), we consider two extreme cases. For case A, we assume that the map to be corrected is small, containing up to a few 10 positions, and that the HPBW 


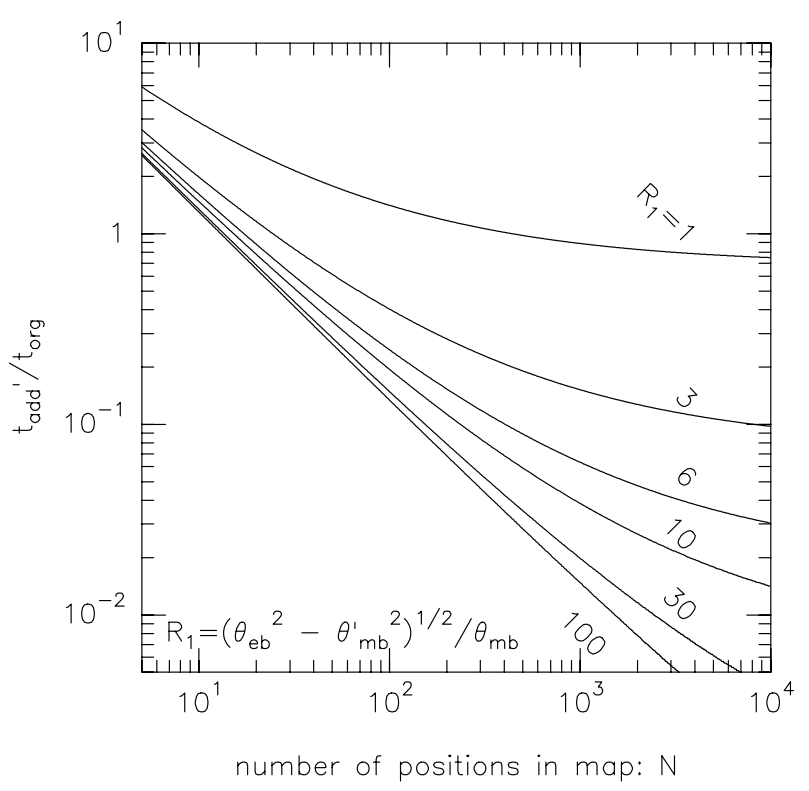

Fig. 1. Time needed for the additional observations (error beam subtraction method): the ratio $t_{\text {add }} / t_{\text {map }}$ (total integration time needed for the additional observations to the integration time of original map) is shown for typical ratios $R_{1}=\left(\theta_{\mathrm{eb}}^{2}-\theta_{\mathrm{mb}}^{\prime 2}\right)^{1 / 2} / \theta_{\mathrm{mb}}=1,3,6,10,30,100$, where $\theta_{\mathrm{eb}}$ and $\theta_{\mathrm{mb}}^{\prime}$ denote the error beam HPBW of the large telescope and main beam HPBW of the small telescope. We choose square geometry for the original map, $a \times a$ (see text for the additional assumptions made)

of the error beam is substantially larger than the main beam HPBW of the small telescope $\left(N^{1 / 2} / 2 R_{1} \ll 1\right)$. For case $\mathrm{B}$ we consider large maps with more than 1000 positions, and the case that the small telescope's main and the large telescope's error beam closely match, both giving $N^{1 / 2} / 2 R_{1} \gg 1$.

For case A, we obtain $t_{\text {add }}^{\prime} / t_{\text {map }} \approx(12.9 / N)$. Thus, the time needed for the additional observations is of the same order than the time needed to complete the high resolution observations to be corrected. For case $\mathrm{B}$, the additional time needed is $t_{\text {add }}^{\prime} / t_{\text {map }} \approx 0.7 / R_{1}^{2}$. This is much smaller than unity, unless $\theta_{\mathrm{eb}} \approx \theta_{\mathrm{mb}}^{\prime}$. In most cases, however, the HPBW of the error beam is larger and the time needed for the additional observations is only a small fraction of the time needed to complete the original map.

\section{Error beam correction by de-convolution}

An alternative correction is the de-convolution of the error beam pattern in Fourier space (de-convolution method). This is motivated by the convolution theorem which links the convolution in the spatial domain to a multiplication of the Fourier transform quantities. The Fourier transform of Eq. (2) reads

$\tilde{T}_{\mathrm{mb}, \mathrm{c}}\left(k_{x}, k_{y}\right)=\tilde{T}_{\mathrm{mb}, \mathrm{c}}=\frac{F_{\mathrm{eff}}}{B_{\mathrm{eff}}} T_{\mathrm{b}} \frac{\left(\tilde{P}_{\mathrm{mb}} \cdot \tilde{S}\right)}{\Omega_{2 \pi}}$, where $k_{x}, k_{y}$ are wave numbers. We use the "tilde" symbol to denote the Fourier transform. With the Fourier transform of Eq. (1), $\tilde{T}_{\mathrm{A}}^{*}=T_{\mathrm{b}}(\tilde{P} \tilde{S}) / \Omega_{2 \pi}$, we obtain

$\tilde{T}_{\mathrm{mb}, \mathrm{c}}=\frac{F_{\mathrm{eff}}}{B_{\mathrm{eff}}} \tilde{T}_{\mathrm{A}}^{*} \frac{\tilde{P}_{\mathrm{mb}}}{\tilde{P}}$,

where $\tilde{T}_{\mathrm{A}}^{*}=\tilde{T}_{\mathrm{A}}^{*}\left(k_{x}, k_{y}\right)$ is the Fourier transform of the observed map. Back transform of Eq. (11) gives the corrected map.

\subsection{Requirements for the de-convolution method}

The de-convolution method requires the observations to be fully sampled, covering a rectangular area. Padding with zeros in order to fill a rectangular map is allowed, provided that the full spatial extent of the emission is observed for the given noise limit. Often, this is not the case, and the error beam de-convolution requires additional, fully sampled observations of the intensity distribution outside the considered map with the same, large telescope (note, that it is the intrinsic property of the error beam to smear the emission to a larger area). For these observations, a larger rms noise and thus a smaller integration time per position is sufficient, because only the integral intensity on the angular scale of the error beam is of interest.

For a quantification we use the same notation and assumptions as above. The original map contains $N$ spectra ( rms noise $\Delta T_{\mathrm{A}}^{*}$ ) and covers an area of $a \times b$. Additional observations are made with the same telescope to extend the existing map: $(a+2 \Delta) \times(b+2 \Delta)$. Here, $\Delta$ is given by the same equation as $\Delta^{\prime}$ above (Eq. 7) with $\theta_{\mathrm{mb}}^{\prime}$ being replaced by $\theta_{\mathrm{mb}}$,

$\Delta \gtrsim\left[\frac{\Theta^{2}}{4 \ln 2} \ln \left(\frac{S N R}{\sqrt{2 \delta_{\mathrm{s}}}} \frac{B_{\mathrm{eb}}}{B_{\mathrm{eff}}}\right)\right]^{1 / 2}$,

and where $\Theta^{2}=\left(\theta_{\mathrm{eb}}^{2}-\theta_{\mathrm{mb}}^{2}\right)$ is the difference of the squared HPBW of the large telescope's main and error beam. Because of $\theta_{\mathrm{mb}}<\theta_{\mathrm{mb}}^{\prime}, \Delta$ is larger than $\Delta^{\prime}$.

Compared to the spectra in the original map, a larger rms noise $\left(\Delta T_{\mathrm{A}, \text { add }}^{*}>\Delta T_{\mathrm{A}}^{*}\right)$ and thus a shorter integration time is sufficient for the spectra in the extended map. In the following, we give an estimate for $\Delta T_{\mathrm{A}}^{*}$,add . Using $\tilde{P}=$ $\tilde{P}_{\mathrm{mb}}+\tilde{P}_{\mathrm{eb}}$ in Eq. (11) we obtain

$\tilde{T}_{\mathrm{mb}, \mathrm{c}}=\frac{F_{\mathrm{eff}}}{B_{\mathrm{eff}}} \tilde{T}_{\mathrm{A}}^{*} \frac{1}{1+\left(\tilde{P}_{\mathrm{eb}} / \tilde{P}_{\mathrm{mb}}\right)}$,

where $\tilde{P}_{\mathrm{eb}} / \tilde{P}_{\mathrm{mb}}$ is given by

$\frac{\tilde{P}_{\mathrm{eb}}}{\tilde{P}_{\mathrm{mb}}}=\frac{B_{\mathrm{eb}}}{B_{\mathrm{mb}}} \exp \left[-\frac{\pi^{2}\left(\theta_{\mathrm{eb}}^{2}-\theta_{\mathrm{mb}}^{2}\right)}{4 \ln 2} k^{2}\right]$,

with $k^{2}=k_{x}^{2}+k_{y}^{2}$. Using $(1+x)^{-1} \approx 1-x$ for small $x=\tilde{P}_{\mathrm{eb}} / \tilde{P}_{\mathrm{mb}} \gtrsim 0$ gives a lower limit to $\tilde{T}_{\mathrm{mb}, \mathrm{c}}$,

$\tilde{T}_{\mathrm{mb}, \mathrm{c}} \gtrsim \frac{F_{\text {eff }}}{B_{\text {eff }}} \tilde{T}_{\mathrm{A}}^{*}\left(1-\frac{B_{\mathrm{eb}}}{B_{\text {eff }}} \exp \left[-\frac{\pi^{2} \Theta^{2} k^{2}}{4 \ln 2}\right]\right)$. 
The back Fourier transform of Eq. (13) then reads

$T_{\mathrm{mb}, \mathrm{c}}>\frac{F_{\mathrm{eff}}}{B_{\mathrm{eff}}}\left(T_{\mathrm{A}}^{*}-\frac{B_{\mathrm{eb}}}{B_{\mathrm{eff}}}\left\langle T_{\mathrm{A}}^{*}\right\rangle_{\mathrm{eb}}\right)$,

where $\left\langle T_{\mathrm{A}}^{*}\right\rangle_{\mathrm{eb}}$ denotes the map observed with the large telescope, smoothed to the angular resolution of error beam. For the spectra in the smoothed map, observations of the original map (rms noise $\Delta T_{\mathrm{A}}^{*}$ ) and the observations in the extended map (rms noise $\Delta T_{\mathrm{A} \text {,add }}^{*}$ ) are averaged. Because of $\Delta T_{\mathrm{A} \text {,add }}^{*}>\Delta T_{\mathrm{A}}^{*}$, an upper limit to the rms noise of the corrected spectra is given by

$\Delta T_{\mathrm{mb}, \mathrm{c}}^{*}<\frac{F_{\mathrm{eff}}}{B_{\mathrm{eff}}}\left[\left(\Delta T_{\mathrm{A}}^{*}\right)^{2}+\frac{B_{\mathrm{eb}}^{2}}{B_{\mathrm{eff}}^{2}}\left\langle\Delta T_{\mathrm{A}, \text { add }}^{*}\right\rangle_{\mathrm{eb}}^{2}\right]^{1 / 2}$.

This is identical to Eq. (A.1) where $\theta_{\mathrm{mb}}^{\prime}, B_{\text {eff }}^{\prime}, F_{\text {eff }}^{\prime}$ are replaced by the quantities of the large telescope, $\theta_{\mathrm{mb}}, B_{\mathrm{eff}}, F_{\text {eff. }}$. Therefore, we can use Eq. (A.6) to obtain

$\Delta T_{\mathrm{A}, \text { add }}^{*} \leq \sqrt{\frac{\pi \delta_{\mathrm{n}}}{\ln 2}} \frac{B_{\mathrm{eff}}}{B_{\mathrm{eb}}} \frac{\Theta}{\Delta x} \Delta T_{\mathrm{A}}^{*}$,

where $\Delta x$ is the sampling of the observations.

\subsection{Time estimate for the additional observations}

The time needed to obtain the additional observations with the large telescope is given by

$t_{\text {add }}^{\prime} \propto \frac{N_{\text {add }}}{\left(\Delta T_{\mathrm{A}}^{*}\right)^{2}}=\left[\frac{2\left(a \Delta+b \Delta+2 \Delta^{2}\right)}{\left(\theta_{\mathrm{mb}} / 2\right)^{2}}\left(\Delta T_{\mathrm{A}, \text { add }}^{*}\right)^{-2}\right]$,

assuming a sampling of $\Delta x=0.5 \theta_{\mathrm{mb}}$ for all observations.

For an evaluation of Eq. (15), we use Eqs. $(12,14)$ and the assumptions made in the previous section $(a=b$, $t_{\text {map }} \propto N\left(\Delta T_{\mathrm{A}}^{*}\right)^{-2}$, same atmospheric conditions for all observations). This gives

$$
\begin{aligned}
t_{\mathrm{add}} / t_{\mathrm{map}} & =\frac{(4 \ln 2)^{1 / 2}}{\pi \delta_{\mathrm{n}}}\left(\frac{B_{\mathrm{eb}}}{B_{\mathrm{mb}}}\right)^{2} N^{-1}\left(\ln \left(\frac{S N R}{\left(2 \delta_{\mathrm{s}}\right)^{1 / 2}} \frac{B_{\mathrm{eb}}}{B_{\mathrm{mb}}}\right)\right)^{1 / 2} \\
& \times\left[\frac{a}{\Theta}+\left(\frac{1}{4 \ln 2} \ln \left(\frac{S N R}{\left(2 \delta_{\mathrm{s}}\right)^{1 / 2}} \frac{B_{\mathrm{eb}}}{B_{\mathrm{mb}}}\right)\right)^{1 / 2}\right] .
\end{aligned}
$$

For a numerical example we adopt $\delta_{\mathrm{s}}, \delta_{\mathrm{n}}=0.02$, a signal-

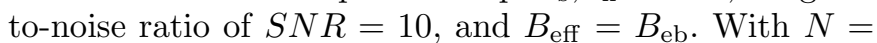
$a^{2} /\left(\theta_{\mathrm{mb}} / 2\right)^{2}$, this gives

$t_{\text {add }} / t_{\text {map }}=52.42 N^{-1}\left[\frac{N^{1 / 2}}{2 R_{2}}+1.19\right]$,

where $R_{2}=\left[\left(\theta_{\mathrm{eb}} / \theta_{\mathrm{mb}}\right)^{2}-1\right]^{1 / 2} \approx \theta_{\mathrm{eb}} / \theta_{\mathrm{mb}}$. The latter approximation can be done because of the angular size of the error beam pattern, typically being $\theta_{\mathrm{eb}} / \theta_{\mathrm{mb}} \approx 15 \ldots 80$. In Fig. 2, the ratio $t_{\text {add }} / t_{\text {map }}$ is shown as a function of $N$ and ratios $10 \leq R_{2} \leq 100$.

If we consider the limit of small maps with up to a few 10 positions $\left(N^{1 / 2} / 2 R_{2} \ll 1\right.$, case $\mathrm{A}$ introduced in Sect. 4.2$)$ we obtain $t_{\text {add }} / t_{\text {map }} \approx 62.3 / N$. Here, the time needed for the additional observations is comparable to

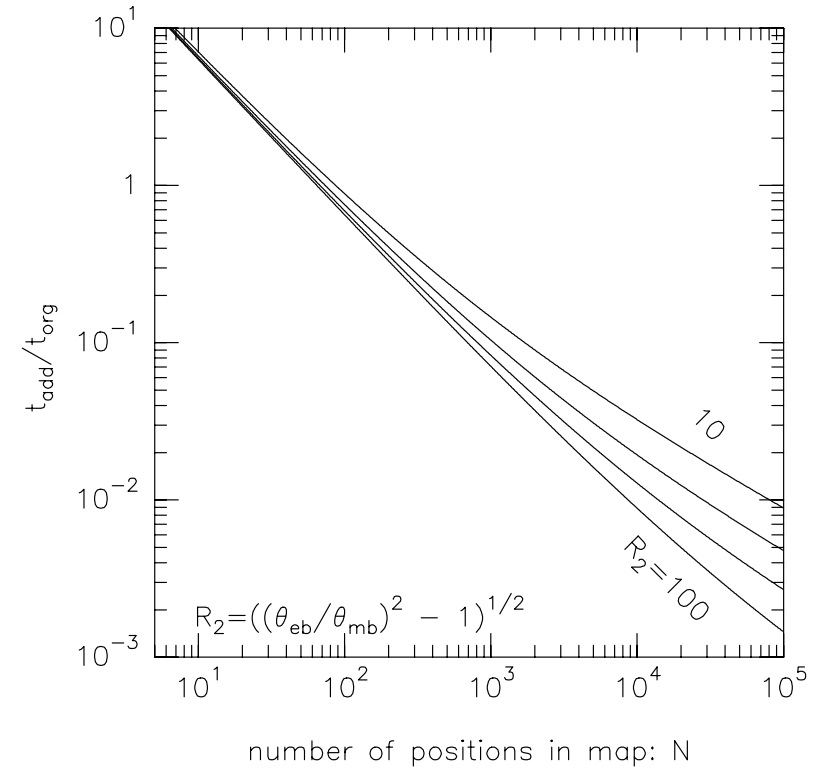

Fig. 2. Time needed for the additional observations (error beam de-convolution method): the ratio $t_{\text {add }} / t_{\text {map }}$ is shown for ratios $R_{2}=\left(\left(\theta_{\mathrm{eb}} / \theta_{\mathrm{mb}}\right)^{2}-1\right)^{1 / 2}=10,20,40,100$. $\theta_{\mathrm{eb}}$ and $\theta_{\mathrm{mb}}$ denote the error beam and main beam HPBW of the large telescope

and may even exceed the time needed to complete the observations for the original map. In the limit of large maps $\left(N^{1 / 2} / 2 R_{2} \gg 1\right.$, case B) we get $t_{\text {add }} / t_{\text {map }}=26.2 / R_{2}$, which is much smaller than $1 \%$ in most cases, because $\left(N^{1 / 2} R_{2}\right) \gtrsim 100$.

\section{Comparison of both correction methods}

\subsection{Observing time required for the additional observations}

For the subtraction method, an area of $\left(a+2 \Delta^{\prime}\right) \times\left(b+2 \Delta^{\prime}\right)$ has to be covered with fully sampled observations made with a small telescope, whereas the de-convolution method requires the existing map to be spatially extended to cover the (larger) area $(a+2 \Delta) \times(b+2 \Delta)$, provided that the observations of the original map are fully sampled. Assuming the same equipment and atmospheric conditions at both telescopes, the time required for the additional observations, $t_{\text {add }}^{\prime} \propto N_{\text {add }}^{\prime}\left(\Delta T_{\mathrm{A}}^{* \prime}\right)^{-2}$ (subtraction method) and $t_{\text {add }} \propto N_{\text {add }}\left(\Delta T_{\text {A,add }}^{*}\right)^{-2}($ de-convolution method $)$, compare as

$\frac{t_{\mathrm{add}}^{\prime}}{t_{\mathrm{add}}}=\frac{\left(a+2 \Delta^{\prime}\right)\left(b+2 \Delta^{\prime}\right)}{2 \Delta(a+b)+4 \Delta^{2}}\left[\frac{F_{\mathrm{eff}}^{\prime}}{F_{\mathrm{eff}}} \frac{B_{\mathrm{eff}}}{B_{\mathrm{eff}}^{\prime}} \frac{\Theta}{\Theta^{\prime}}\right]^{2}$,

where we have used Eqs. (8) and (14) for $\Delta T_{\mathrm{A}}^{* \prime}$ and $\Delta T_{\mathrm{A}, \text { add }}^{*}$. Recall that $\Theta^{2}=\theta_{\mathrm{eb}}^{2}-\theta_{\mathrm{mb}}^{2} \approx \theta_{\mathrm{eb}}^{2}$ and $\Theta^{\prime 2}=$ $\theta_{\mathrm{eb}}^{2}-\theta_{\mathrm{mb}}^{\prime 2}$ are the differences between the squared HPBW of the Gaussian error beam $\left(\theta_{\mathrm{eb}}\right)$ and the main beam of the large (small) telescope $\theta_{\mathrm{mb}}\left(\theta_{\mathrm{mb}}^{\prime}\right)$. 
For small maps $\left(a, b \ll 2 \Delta, 2 \Delta^{\prime}\right.$, discussed in Sect. 4.2 as case A) we obtain

$\frac{t_{\mathrm{add}}^{\prime}}{t_{\mathrm{add}}} \approx\left[\frac{F_{\mathrm{eff}}^{\prime}}{F_{\mathrm{eff}}} \frac{B_{\mathrm{eff}}}{B_{\mathrm{eff}}^{\prime}}\right]^{2}$,

and thus that the subtraction method is more efficient. The additional observations with the smaller telescope are less time consuming $\left(B_{\text {eff }}<B_{\text {eff }}^{\prime}\right.$, while $\left.F_{\text {eff }} \approx F_{\text {eff }}^{\prime}\right)$. For spatially extended maps $\left(a, b \gg \Delta, \Delta^{\prime}\right.$; case $\left.\mathrm{B}\right)$ we get

$\frac{t_{\mathrm{add}}^{\prime}}{t_{\mathrm{add}}} \approx \frac{a b}{2 \Delta(a+b)}\left[\frac{F_{\mathrm{eff}}^{\prime}}{F_{\mathrm{eff}}} \frac{B_{\mathrm{eff}}}{B_{\mathrm{eff}}^{\prime}} \frac{\Theta}{\Theta^{\prime}}\right]^{2}$.

Typically, we have $\theta_{\mathrm{mb}} \ll \theta_{\mathrm{mb}}^{\prime} \lesssim \theta_{\mathrm{eb}}$, and hence $\Theta / \Theta^{\prime}>1$. With $B_{\text {eff }} / B_{\text {eff }}^{\prime} \lesssim 1$, this gives $t_{\text {add }}^{\prime} / t_{\text {add }}>1$, and that the additional observations with the large telescope are less time consuming. For large maps and the de-convolution method, the disadvantage of the smaller main beam efficiency $\left(B_{\text {eff }}<B_{\text {eff }}^{\prime}\right)$ is outweighed by the smaller area which has to be covered with the additional observations.

\subsection{Limitations of the correction methods}

Limitations of the error beam subtraction method are given by the accuracy of the intensity calibration of the observations made with the smaller telescope. For instance, they potentially suffer from a significant error beam pick-up, too. However, this is a second order effect in most cases, and the subtraction method still gives a better estimate for the intensity detected by the main beam than the commonly used antenna temperature and main beam brightness temperature. For the KOSMA observations (used to correct the IRAM key-project CO $J=2 \rightarrow 1$ data) we estimate the error beam pick-up to be smaller than $18 \%$. This is to be compared to an error beam pick-up in the key-project maps of 31-50\% (Paper III).

An additional limitation is given by the finite angular resolution of observations made with the small telescope. This might be a problem for the correction of observations made with large (sub-)mm telescopes (diameter of several ten meters). Here, large-scale deformations in the reflector surface potentially result in an error beam with a small HPBW, such as the first error beam of the IRAM $30 \mathrm{~m}$ $\left(\theta_{\mathrm{eb}} \approx 10 \theta_{\mathrm{mb}}\right.$, see Greve et al. 1998). A correspondingly large telescope with a clean beam pattern is then needed for a correction, which may be difficult to find.

For the de-convolution method, additional observations have to be made for a large number of positions with an extremely short integration time per position (only the intensity integrated on the angular extent of the error beam pattern is of interest). This possibly results in an huge overhead in dead time due to telescope motion, data acquisition, etc. In addition, the large number of spectra in the extended map creates a comparably large amount of raw data to be handled in a relatively short time. The application of de-convolution method therefore may be impracticable or even impossible to apply. For an illustration, we give the example of the ${ }^{12} \mathrm{CO} 2 \rightarrow 1$ map of MCLD
$123.5+24.9$, observed in the framework of the IRAM keyproject. The correction for the pick-up in the second error beam requires additional observations with the IRAM $30 \mathrm{~m}$ at $\sim 15000$ positions and an integration time of less than $0.1 \mathrm{~s}$ per position. Even more extreme numbers are obtained for the correction of the third error beam. This is currently not feasible and we apply the subtraction method to correct the key-project data.

\section{Summary}

The presence of a significant error beam pattern potentially gives rise to additional pick-up of intensity from outside the main beam, in particular for observations of spatially extended sources. For (sub-)mm observations suffering from a significant error beam pick-up we introduce the corrected main beam brightness temperature, $T_{\mathrm{mb}, \mathrm{c}}$ as new intensity scale. It provides a better estimate for the intensity detected by the main beam than the commonly used main beam brightness temperature and antenna temperature. In the limit of a negligible error beam pick-up, the corrected main beam brightness temperature is equal to the main beam brightness temperature.

We discuss and compare two different correction methods in detail. The subtraction method, which uses additional observations made with a smaller telescope, and the de-convolution method, where the error beam pick-up is de-convolved from the observed map in the Fourier space. Both methods have been described and successfully applied to observed spectral line maps (cf. Kalberla et al. 1980; Dame \& Thaddeus 1994; Hartmann et al. 1996; Schneider et al. 1998).

The de-convolution method is attractive for the correction of fully sampled maps which cover the full spatial extent of the emission for the given noise limit. Here, the observed map contains all information required to correct for the error beam pick-up. For maps which cover only a fraction of a more extended intensity distribution, both correction methods require additional observations. Observations with a smaller telescope are required for the subtraction method, while the existing (fully sampled) map has to be extended for the de-convolution method. Typically, the additional observations require less time than was needed to complete the original observations to be corrected (in terms of total integration time), except for small maps with a few ten positions. For large maps ( $\gtrsim 10^{4}$ positions), such as those typically observed with large surveys, the time needed is only a few percent of the time spent for the original map. Though this a minor fraction, it should be taken into account when planning large mapping projects and a corresponding fraction of the time should be allocated for the additional observations required.

For the correction of a small map or single spectra, the subtraction method is favorable because the additional observations can be done more efficiently with a smaller telescope (in this context, "small" means that the angular extent of the original map is smaller than a few times 
$\left(\theta_{\mathrm{eb}}^{2}-\theta_{\mathrm{mb}}^{\prime 2}\right)^{1 / 2}$, where $\theta_{\mathrm{eb}}$ is the HPBW of the error beam and $\theta_{\mathrm{mb}}^{\prime}$ is the HPBW of the small telescope). The $s u b$ traction method is limited by a potentially significant error beam pick-up in the observations made with the smaller telescope and the lower angular resolution. In particular, one has to consider that smaller telescopes tend to have cleaner beams allowing for a more accurate correction of the error beam pick-up, but possibly do not have the required angular resolution.

For the correction of large maps (angular extent $\gg$ error beam), the de-convolution method is attractive because the integration time for the additional observations is smaller than for the subtraction method. In practice, however, these observations are often limited by the data handling capacity and dead time (as a large amount of data has to be collected in a relatively short time) and by the limited availability of large (sub-)mm telescope for such (additional) observations.

Acknowledgements. The KOSMA $3 \mathrm{~m}$ radio telescope at Gornergrat-Süd Observatory is operated by the University of Cologne and supported by the Deutsche Forschungsgemeinschaft through grant SFB-301, as well as special funding from the Land Nordrhein-Westfalen. The receiver development was partly funded by the Bundesminister für Forschung and Technologie. The Observatory is administered by the Internationale Stiftung Hochalpine Forschungsstationen Jungfraujoch und Gornergrat, Bern. The authors have benefitted from a joint European grant (Procope, grant\# 312-probmbw-gg) during the years 1995 and 1996. This research made use of NASA's Astrophysics Data System Abstract Service.

\section{Appendix A: Error beam correction by using observations of a smaller telescope: Influence of edge effects and of the noise in the observations obtained with the smaller telescope}

Consider a large telescope with the beam pattern denoted by $P$ and the normalization $P(0,0)=1$. We assume that the beam pattern consists of a tapered Airy pattern $\mathcal{A}_{\mathrm{T}}$, approximated by a Gaussian of half power beam width (HPBW) $\theta_{\mathrm{mb}}$, and a Gaussian error beam $P_{\mathrm{eb}}$ of HPBW $\theta_{\mathrm{eb}}: P=\mathcal{A}_{\mathrm{T}}+P_{\mathrm{eb}}$. Forward and main beam efficiency of the telescope are denoted by $F_{\text {eff }}$ and $B_{\text {eff }}$, and the "efficiency" of the Gaussian error beam is defined according $B_{\text {eb }}=\iint P_{\text {eb }} \mathrm{d} \Omega / \Omega_{4 \pi}$.

A spatially extended source is observed and the resulting map covers a rectangular area $a \times b$. The error beam correction is done using the subtraction method. Additional observations are made with a small telescope (main beam HPBW and efficiency of $\theta_{\mathrm{mb}}^{\prime}, B_{\mathrm{eff}}^{\prime}$, forward efficiency $\left.F_{\text {eff }}^{\prime}\right)$. They cover a larger area $\left(a+2 \Delta^{\prime}\right) \times(b+$ $\left.2 \Delta^{\prime}\right)$ because of the angular extent of the error beam, $\theta_{\mathrm{eb}} \gtrsim \theta_{\mathrm{mb}}^{\prime}$.

The error beam pick-up is estimated using Eq. (6), with the summation being done over only one Gaussian error beam. The corrected main beam brightness temperature is then approximated by

$T_{\mathrm{mb}, \mathrm{c}} \approx \frac{F_{\mathrm{eff}}}{B_{\mathrm{eff}}}\left[T_{\mathrm{A}}^{*}-T_{b} \frac{B_{\mathrm{eb}}}{F_{\mathrm{eff}}}\left\langle T_{\mathrm{mb}}^{\prime}\right\rangle_{\mathrm{eb}}\right]$.

The accuracy of the corrected main beam brightness temperature $\left(\Delta T_{\mathrm{mb}, \mathrm{c}}\right)$ is given by the rms noise in the original observations $\left(\Delta T_{\mathrm{A}}^{*}\right)$ and the noise in the additional observations made with the smaller telescope. An additional, systematic error might be due to the limited spatial extent of the map observed with the smaller telescope, missing a fraction of the error beam. Although being a systematic error, we include the latter as a Gaussian average in our error calculations, as if it is an independent, statistical error.

With the accuracy of the estimated error beam pickup denoted by $\Delta\left\langle T_{\mathrm{mb}}^{\prime}\right\rangle_{\mathrm{eb}}$, we deduce the accuracy of the corrected main beam brightness temperature scale to be

$\Delta T_{\mathrm{mb}, \mathrm{c}}=\frac{F_{\mathrm{eff}}}{B_{\mathrm{eff}}}\left(\Delta T_{\mathrm{A}}^{*}\right)\left[1+\left(\frac{B_{\mathrm{eb}}}{F_{\mathrm{eff}}} \frac{\Delta\left\langle T_{\mathrm{mb}}^{\prime}\right\rangle_{\mathrm{eb}}}{\Delta T_{\mathrm{A}}^{*}}\right)^{2}\right]^{1 / 2}$,

where we neglect a possible error beam pick-up in the observations of the smaller telescope.

For a reliable error beam correction, the additional error introduced by the rms noise and the finite size of the observations made with the small telescope should be small compared to the rms noise in the original observations, $\Delta\left\langle T_{\mathrm{mb}}^{\prime}\right\rangle \ll \Delta T_{\mathrm{A}}^{*}$. Therefore, we can assume $x=\left(\frac{B_{\text {eb }}}{F_{\text {eff }}} \frac{\Delta\left\langle T_{\mathrm{mb}}^{\prime}\right\rangle_{\mathrm{eb}}}{\Delta T_{\mathrm{A}}^{*}}\right)^{2} \ll 1$ which allows us to use the approximation $(1+x)^{1 / 2} \approx 1+\frac{1}{2} x$ to obtain

$\Delta T_{\mathrm{mb}, \mathrm{c}} \approx \frac{F_{\mathrm{eff}}}{B_{\mathrm{eff}}}\left(\Delta T_{\mathrm{A}}^{*}\right)\left[1+\frac{1}{2} x\right]$.

Specifying the relative error introduced by the error beam correction as $\delta_{\mathrm{eb}}$, we can write

$\frac{1}{2} x=\frac{1}{2}\left(\frac{B_{\mathrm{eb}}}{B_{\mathrm{eff}}^{\prime}} \frac{F_{\mathrm{eff}}^{\prime}}{F_{\mathrm{eff}}} \frac{\Delta\left\langle T_{\mathrm{A}}^{* \prime}\right\rangle_{\mathrm{eb}}}{\Delta T_{\mathrm{A}}^{*}}\right)^{2}<\delta_{\mathrm{eb}}$,

which gives an upper limit

$\Delta\left\langle T_{\mathrm{A}}^{* \prime}\right\rangle_{\mathrm{eb}}<\sqrt{2 \delta_{e b}}\left(\frac{F_{\mathrm{eff}}}{F_{\mathrm{eff}}^{\prime}} \frac{B_{\mathrm{eff}}^{\prime}}{B_{\mathrm{eb}}}\right)\left(\Delta T_{\mathrm{A}}^{*}\right)$

\section{A.1. Edge effects and minimum map size for the observations made with the smaller telescope}

We now determine the systematic error introduced due to the finite spatial extent of the map observed with the smaller telescope, $\left(a+2 \Delta^{\prime}\right) \times\left(b+2 \Delta^{\prime}\right)$, and require that it is small compared to the rms noise in the original spectra, $\Delta T_{\mathrm{A}}^{*}$. For an estimate, we assume an uniform source distribution of an angular extent much larger than the error beam pattern. Dividing Eq. (A.2) by $T_{\mathrm{A}}^{*}$ gives

$\frac{\Delta\left\langle T_{\mathrm{A}}^{* \prime}\right\rangle_{\mathrm{eb}}}{T_{\mathrm{A}}^{*}}<\sqrt{2 \delta_{\mathrm{s}}}\left(\frac{F_{\mathrm{eff}}}{F_{\mathrm{eff}}^{\prime}} \frac{B_{\mathrm{eff}}^{\prime}}{B_{\mathrm{eb}}}\right) \frac{\Delta T_{\mathrm{A}}^{*}}{T_{\mathrm{A}}^{*}}$, 
where we have used that $T_{\mathrm{A}}^{*}=\left(B_{\mathrm{eff}} / F_{\mathrm{eff}}\right) T_{\mathrm{mb}}$. Typically, $\delta_{\mathrm{s}}$ is of the order of a few percent. The assumption of a uniform source distribution of angular extent larger than the error beam pattern gives $T_{\mathrm{A}}^{*}=T_{\mathrm{A}}^{* \prime}$. Thus, $\Delta\left\langle T_{\mathrm{A}}^{* \prime}\right\rangle_{\mathrm{eb}} / T_{\mathrm{A}}^{*}$ can be re-written to

$\frac{\Delta\left\langle T_{\mathrm{A}}^{* \prime}\right\rangle_{\mathrm{eb}}}{T_{\mathrm{A}}^{*}}=\frac{\Delta\left\langle T_{\mathrm{A}}^{* \prime}\right\rangle_{\mathrm{eb}}}{T_{\mathrm{A}}^{* \prime}} \lesssim \frac{\iint_{r}^{\Delta^{\prime}} G_{\Theta^{\prime}}(r) \mathrm{d} x \mathrm{~d} y}{\iint_{r}^{\infty} G_{\Theta^{\prime}}(r) \mathrm{d} x \mathrm{~d} y}$,

where $\Delta\left\langle T_{\mathrm{A}}^{* \prime}\right\rangle_{\mathrm{eb}} / T_{\mathrm{A}}^{*}$ is the fraction of the error beam pick-up which is missed. $G_{\Theta^{\prime}}$ is the normalized, 2-dimensional Gaussian of HPBW $\Theta^{\prime}, \quad G_{\Theta^{\prime}}(r)=$ $\left(4 \ln 2 / \Theta^{\prime 2} \pi\right) \exp \left[-4 \ln 2\left(r / \Theta^{\prime}\right)^{2}\right]$, with $\Theta^{\prime 2}=\theta_{\mathrm{eb}}^{2}-\theta_{\mathrm{mb}}^{\prime 2}$ and $r=\left(x^{2}+y^{2}\right)^{1 / 2}$. Evaluation of the integrals gives

$\frac{\Delta\left\langle T_{\mathrm{A}}^{* \prime}\right\rangle_{\mathrm{eb}}}{T_{\mathrm{A}}^{* \prime}} \approx \exp \left[-4 \ln 2\left(\Delta^{\prime} / \Theta^{\prime}\right)^{2}\right]$

which provides an upper limit to $\Delta\left\langle T_{\mathrm{A}}^{* \prime}\right\rangle_{\mathrm{eb}} / T_{\mathrm{A}}^{*}$. This finally gives

$\Delta^{\prime}<\left[\frac{\Theta^{\prime 2}}{4 \ln 2} \ln \left(\frac{S N R}{\sqrt{2 \delta_{\mathrm{s}}}} \frac{F_{\mathrm{eff}}^{\prime}}{F_{\mathrm{eff}}} \frac{B_{\mathrm{eb}}}{B_{\mathrm{eff}}^{\prime}}\right)\right]^{1 / 2}, \quad \theta_{\mathrm{eb}} \geq \theta_{\mathrm{mb}}^{\prime}$,

where $S N R=T_{\mathrm{A}}^{*} / \Delta T_{\mathrm{A}}^{*}$ is the signal-to-noise ratio of the observations made with the large telescope. For typical values $\left(F_{\text {eff }}^{\prime} / F_{\text {eff }} \approx 1, B_{\text {eb }} / B_{\text {eff }}^{\prime} \sim 0.1-0.5, \delta_{\mathrm{s}} \sim\right.$ 0.02 and SNR of a few 10 ) we obtain $\Delta^{\prime} \approx 1 \ldots 2 \Theta^{\prime}$. In the limit of equal HPBW for the small telescope's main and the large telescope's error beam $\left(\theta_{\mathrm{eb}}=\theta_{\mathrm{mb}}^{\prime}\right)$ we have $\Theta^{\prime}=0$ and $\Delta^{\prime}$ vanishes.

\section{A.2. Contribution of the noise in the observations of the smaller telescope to the noise in the corrected spectra}

To estimate the additional noise introduced with the error beam correction, we assume that the additional observations are made on a regular grid, and that $T_{\mathrm{A}}^{* \prime}(x, y)$ gives the observed intensity as function of the offset position $x, y$. The spectrum at position $\left(x_{0}, y_{0}\right)$, smoothed to the resolution of the error beam, is then given by

$$
\left\langle T_{\mathrm{A}}^{* \prime}\right\rangle_{\mathrm{eb}}=\frac{\sum_{x, y} G_{\Theta^{\prime}}\left(r-r_{0}\right) T_{\mathrm{A}}^{* \prime}(x, y)}{\sum_{x, y} G_{\Theta^{\prime}}\left(r-r_{0}\right)}, \quad \Theta^{\prime}>0 .
$$

Here, $G_{\Theta^{\prime}}\left(r-r_{0}\right)$ is the 2-dimensional Gaussian defined above with $r-r_{0}=\left(\left(x-x_{0}\right)^{2}-\left(y-y_{0}\right)^{2}\right)^{1 / 2}$. The summation is done over all positions $(x, y)$ of the grid. For $\Theta^{\prime}=0$ (the main beam HPBW of the small telescope matches the HPBW of the error beam) we have $\left\langle T_{\mathrm{A}}^{* \prime}\right\rangle_{\mathrm{eb}}=T_{\mathrm{A}}^{* \prime}$. The rms noise of the smoothed spectra is given by

$\left\langle\Delta T_{\mathrm{A}}^{* \prime}\right\rangle_{\mathrm{eb}}=\frac{\left[\sum_{x, y}\left[G_{\Theta^{\prime}}\left(r-r_{0}\right) \Delta T_{\mathrm{A}}^{* \prime}(x, y)\right]^{2}\right]^{1 / 2}}{\sum_{x, y} G_{\Theta^{\prime}}(r)}, \Theta^{\prime}>0$,

and $\left\langle\Delta T_{\mathrm{A}}^{* \prime}\right\rangle_{\mathrm{eb}}=\Delta T_{\mathrm{A}}^{* \prime}(x, y)$ for $\Theta^{\prime}=0$. We assume a constant rms noise level, $\Delta T_{\mathrm{A}}^{* \prime}(x, y)=\Delta T_{\mathrm{A}}^{* \prime}$ for the observations made with the small telescope. Multiplication of the numerator and denominator by the sampling $\Delta x \Delta y$ and replacing the summation by an integration gives

$\left\langle\Delta T_{\mathrm{A}}^{* \prime}\right\rangle_{\mathrm{eb}} \approx \Delta T_{\mathrm{A}}^{* \prime} \frac{\left[\iint\left[G_{\Theta^{\prime}}(r)\right]^{2} \mathrm{~d} x \mathrm{~d} y\right]^{1 / 2}(\Delta x \Delta y)^{1 / 2}}{\iint G_{\Theta^{\prime}}(r) \mathrm{d} x \mathrm{~d} y}$.

This includes the limiting case of $\Theta^{\prime} \rightarrow 0$ where the Gaussian approaches the $\delta$-function, $G_{\Theta^{\prime}}(r) \rightarrow \delta(r)$. Evaluation of the integrals for $\Theta^{\prime}>0$ gives

$\left\langle\Delta T_{\mathrm{A}}^{* \prime}\right\rangle_{\mathrm{eb}} \approx \frac{\Delta x^{\prime}}{\Theta^{\prime}} \sqrt{\frac{4 \ln 2}{2 \pi}}\left(\Delta T_{\mathrm{A}}^{* \prime}\right), \quad \Theta^{\prime}>0$.

Thus, the rms noise required for the observations of the small telescope is

$\Delta T_{\mathrm{A}}^{* \prime} \approx \sqrt{\frac{\pi \delta_{\mathrm{n}}}{\ln 2}} \frac{F_{\mathrm{eff}}}{F_{\mathrm{eff}}^{\prime}} \frac{B_{\mathrm{eff}}^{\prime}}{B_{\mathrm{eb}}} \frac{\Theta^{\prime}}{\Delta x^{\prime}}\left(\Delta T_{\mathrm{A}}^{*}\right), \quad \Theta^{\prime}>0$.

Typical values $\left(F_{\mathrm{eff}} / F_{\mathrm{eff}}^{\prime} \approx 1, B_{\mathrm{eff}}^{\prime} / B_{\mathrm{eb}}=0.1 \ldots 0.5\right.$, $\Theta^{\prime} / \Delta x^{\prime} \gtrsim 1$ and $\left.\delta_{\mathrm{n}} \sim 0.02\right)$ give a ratio $\left(\Delta T_{\mathrm{A}}^{* \prime} / \Delta T_{\mathrm{A}}^{*}\right)$ of the order of a few tenths.

\section{References}

Baars, J. W. M. 1973, IEEE Trans. Ant. Propag., AP-21, 461 Bensch, F., Panis, J.-F., Stutzki, J., Heithausen, A., \& Falgarone, E. 2001, A\&A, 365, 275 (Paper III)

Dame, T., \& Thaddeus, P. 1994, ApJ, 436, L173

Downes, D. 1989, Introductory courses in Galaxies (Springer)

Falgarone, E., Panis, J.-F., Heithausen, A., et al. 1998, A\&A, 331, 669 (Paper I)

Garcia-Burillo, S., Guélin, M., \& Cernicharo, J. 1993, A\&A, 274,123

Greve, A., Kramer, C., \& Wild, W. 1998, A\&AS, 133, 271

Harten, R. H. 1973, AJ, 78, 565

Hartmann, D., Kalberla, P. M. W., Burton, W. B., \& Mebold, U. 1996, A\&AS, 119, 115

Heiles, C., \& Hoffman, W. 1968, AJ, 73, 412

Heithausen, A., Bensch, F., Stutzki, J., Falgarone, E., \& Panis, J.-F. 1998, A\&A, 331, L65

Hills, R. E., \& Richter, J. 1992, The JCMT Newsletter, Aug. 24

Kalberla, P. M. W., Mebold, U., \& Reich, W. 1980, A\&A, 82, 275

Kaufmann, P., Abraham, Z., Scalise, E., et al. 1987, IEEE Trans. Ant. Propag., AP-35, 996

Kramer, C. 1997, Calibration of spectral line data at the IRAM $30 \mathrm{~m}$ ratio telescope (v2.1), IRAM report

Kutner, M. L., \& Ulich, B. L. 1981, ApJ, 250, 341

Prestage, R. 1993, The JCMT Newsletter, July 27

Rohlfs, K., \& Wilson, T. L. 1996, Tools of Radio Astronomy, 2nd edition (Springer-Verlag Berlin)

Ruze, J. 1952, Suppl. al Nuovo Cimento, 9, 364

Ruze, J. 1966, Proc. IEEE, 54, 633

Scheffler, H. 1962, Z. f. Astrophys., 55, 1

Schneider, N., Stutzki, J., Winnewisser, G., \& Block, D. 1998, A\&A, 335, 1049

Shifrin, Y. S. 1971, Statistical Antenna Theory (The Golem Press, Boulder, Colorado, USA)

van Woerden, H., Takakkubo, K., \& Braes, L. L. E. 1962, Bulletin of the Astronomical Institutes of the Netherlands, Vol. 16, No. 524, 321

Westerhout, G., Wendlandt, \& Harten, R. H. 1973, AJ, 78, 569 